\title{
Left ventricular mechanical dyssynchrony under stress: Isn't it time to conduct a prospective multicenter study?
}

\author{
Samaneh Salimian, $\mathrm{PhD}^{\mathrm{a}}$ \\ a Department of Biomedical Sciences, University of Montreal, Montreal, QC, Canada
}

Received Dec 28, 2018; accepted Dec 28, 2018

doi: $10.1007 / \mathrm{s} 12350-019-01599-\mathrm{x}$

\section{See related article, pp. 2247-2257}

Synchronicity matters! The loss of contraction synchrony between left ventricular (LV) myocardial segments leads to structural and functional alterations that may eventually result in heart failure. Mechanical dyssynchrony (MD) or disparities in wall contraction timing may be secondary to delayed electric conduction, delayed electromechanical coupling, or changes in regional myocardial properties due to ischemic damage or myocardial infarction. ${ }^{1}$

During the last two decades, many efforts have been made to detect and measure the LVMD accurately, since it has shown promising results in clinical practice. LVMD has presented incremental clinical value in prognostication and risk stratification of different subsets of patients with ischemic ${ }^{2}$ or nonischemic dilated cardiomyopathy, ${ }^{3}$ acute myocardial infarction, ${ }^{4}$ coronary artery disease, ${ }^{5}$ and even in patients with preserved ventricular function without signs or symptoms of heart disease, allowing early intervention before the occurrence of global LV dysfunction. ${ }^{6}$ Also, it has been shown that LVMD plays a role for prognosis after cardiac resynchronization therapy (CRT) implantation. ${ }^{7}$ It is of particular importance because $30 \%$ of patients do not show any beneficial outcome with $\mathrm{CRT}^{1}$ and their condition might even worsen as a result of changes in cardiac mechanics. ${ }^{8}$ However, the efficiency of MD as a screening tool to identify responders to CRT is still

See related article, pp. 2247-2257.

Reprint requests: Samaneh Salimian, PhD, Department of Biomedical

Sciences, University of Montreal, Montreal, QC, Canada; samaneh.salimian@umontreal.ca

J Nucl Cardiol 2020;27:2258-60.

1071-3581/\$34.00

Copyright (C) 2019 American Society of Nuclear Cardiology. under question. In fact, the success of CRT response depends on a complex set of variables which include, but are not limited to, the extent of the viable myocardium in the target segment for pacing, successful delivery of a pacing lead to a segment with the most delayed activation, and the presence and magnitude of LVMD. ${ }^{9}$ It seems that the latter case itself holds two variables based on the condition of MD measurements either at rest or stress.

While limited, much of the literature on the assessment of the effects of physical exercise or pharmacological stress on LVMD relates to the patients with impaired LV function amenable to CRT using different echocardiographic techniques. ${ }^{10}$ Among these studies, there is a general agreement that LVMD is not a stable phenomenon, and its presence and extent may change in response to the level of the stress or exercise. However, these studies have reported different stressinduced responses on LVMD in heart failure patients. ${ }^{11-14}$ These differences may be explained by differences in echo methodologies, defined indexes, and types of stressors used. But, even in a single study carried out on patients with the same pathologies, stress tests have been reported to increase, decrease, or have no effect on LVMD. ${ }^{11}$ A possible explanation for this irregular outcome could be the difference in intrinsic myocardial properties, which are specific to each patient. The association between stress-induced changes on LVMD and the response to CRT has been addressed by some of these studies. Accordingly, stress-induced variation in MD has been shown to have a more significant relationship to clinical outcomes and long-term survival than resting MD following CRT. ${ }^{12,14}$ The robustness of stress MD for the prognosis has also been proved in patients with dilated cardiomyopathy and narrow QRS. ${ }^{15}$ In addition, exercise MD was shown to be a stronger predictor of response to CRT than resting MD. ${ }^{16}$ A question may rise here instantly: Why is the assessment of LVMD for the purpose of selecting CRT 
responders or even for predicting the prognosis of patients still considered only at rest? It might be due to the complexity of MD measurements at stress condition using echocardiography. In fact, echocardiographic methods have widely been used until now for diagnosing LVMD. Identifying the dyssynchrony even at rest has always been challenging using echo techniques because of the absence of standard and automatic MD criteria and due to measurement errors that may happen consequently during the complicated procedures, which, overall, lead to unreliable results. The accuracy of the results would be the worst for under-stress MD assessments because of the heart rate variations. The subjectivity of echo techniques also prevents the development of prospective clinical studies with a multicenter nature. Based on echo-driven rest $\mathrm{MD}$ assessments, the predictive value of MD for selecting the best CRT responders has been criticized, ${ }^{17}$ and it has been suggested to consider MD as a response parameter after CRT rather than a parameter to select patients for CRT. ${ }^{8}$ Although the innovative phase analysis technique in nuclear cardiology has helped to overcome the subjectivity and inaccuracy of echo-driven MD results, it has not been much used for stress MD analyses, and even for rest MD analyses in large clinical trials.

In this issue of the Journal of Nuclear Cardiology, Legallois et al. ${ }^{18}$ have made a contribution to the evaluation of LVMD under stress constituting the first study analyzing the repeatability of LV dyssynchrony assessments derived from SPECT myocardial perfusion imaging (MPI) in a multicenter design. This multicentric study evaluated whether the repeatability of the LV dyssynchrony assessments using SPECT MPI is high enough to allow detecting either a synchrony reserve or significant synchrony changes during low-dose dobutamine infusion in patients with ischemic cardiomyopathy. They retrospectively analyzed data prospectively acquired from 61 patients with LV ejection fraction of $<50 \%$ and ischemic cardiomyopathy who were referred for the evaluation of myocardial viability in 10 different centers. Each patient underwent two consecutive rest MPI SPECT tests 40 to 60 minutes after the injection of a $99 \mathrm{mTc}$-labeled tracer. After the completion of rest acquisitions, they administered a lowdose graded infusion of dobutamine at a starting dose of $5 \mathrm{mcg} / \mathrm{kg} / \mathrm{min}$ during 5 minutes, increasing to $10 \mathrm{mcg} /$ $\mathrm{kg} / \mathrm{min}$ in the absence of clinical or electrical sign of ischemia. Taking into account the short half-life of dobutamine, they initiated under-stress SPECT acquisition after 3 minutes of steady infusion of the target dose. The LVMD was assessed using QGS software through bandwidth (BW), standard deviation, and entropy. They concluded that LVMD and functional assessments using SPECT MPI are quite repeatable in patients with LV dysfunction and ischemia and that entropy has the best repeatability, with a concordance correlation coefficient of 0.8 , among other MD parameters. These results are highly promising since they are derived from a study that is multicentric with the potential of having variability in SPECT systems like scanner sensitivity and spatial resolution. Also, the results relate to the patients with myocardial infarction and LV dysfunction, a clinical condition that changes the radiotracer count intensity and myocardial wall thickening, which may affect the myocardial segmentation and consequent phase analyses. As expected, entropy showed the highest repeatability among other MD parameters since, although a histogram-based approach is used for its estimation, it is less dependent on the shape of the phase distribution histogram. The robustness and sensitivity of entropy in specifying the dyssynchrony variations during the stress SPECT have been reported previously even in healthy canine subjects. ${ }^{19,20}$ In this study, entropy $(P=0.04)$ and BW $(P=0.049)$ indicated a significant contraction synchrony improvement under stress condition in patients with ischemia and depressed ejection fraction. Low-dose dobutamine might enforce higher coordination in regional mechanical contraction in these patients.

While the current study reveals the feasibility of under stress LVMD assessments using MPI SPECT, it opens an avenue to employ this method for better understanding of the role of MD in patient selection for CRT, an area which still remains the most crucial reason for MD assessments. Showing excellent multicentric repeatability for MD assessments using MPI SPECT develops enough motivation for conducting prospective multicenter studies. It is time to show the clinicians, with absolute certainty, whether stress-induced variations in MD can serve as a CRT selection criterion by conducting prospective randomized multicenter trials using stress MPI SPECT.

\section{Disclosures}

The author, Samaneh Salimian, states that she has nothing to disclose.

\section{References}

1. Kirk JA, Kass DA. Electromechanical dyssynchrony and resynchronization of the failing heart. Circ Res 2013;113:765-76.

2. AlJaroudi W, Alraies MC, Hachamovitch R, Jaber WA, Brunken $\mathrm{R}$, Cerqueira MD, et al. Association of left ventricular mechanical dyssynchrony with survival benefit from revascularization: A study of gated positron emission tomography in patients with ischemic LV dysfunction and narrow QRS. Eur J Nucl Med Mol Imaging 2012;39:1581-91. 
3. Kano N, Okumura T, Isobe S, Sawamura A, Watanabe N, Fukaya $\mathrm{K}$, et al. Left ventricular phase entropy: Novel prognostic predictor in patients with dilated cardiomyopathy and narrow QRS. J Nucl Cardiol 2018;25:1677-87.

4. Shin SH, Hung CL, Uno H, Hassanein AH, Verma A, Bourgoun $\mathrm{M}$, et al. Mechanical dyssynchrony after myocardial infarction in patients with left ventricular dysfunction, heart failure, or both. Circulation 2010;121:1096-103.

5. Fudim M, Fathallah M, Shaw LK, Liu PR, James O, Samad Z et al. The prognostic value of diastolic and systolic mechanical left ventricular dyssynchrony among patients with coronary heart disease. JACC Cardiovasc Imaging 2018.

6. Sharma RK, Volpe G, Rosen BD, Ambale-Venkatesh B, Donekal $S$, Fernandes V, et al. Prognostic implications of left ventricular dyssynchrony for major adverse cardiovascular events in asymptomatic women and men: The Multi-Ethnic Study of Atherosclerosis. J Am Heart Assoc 2014;3:e000975.

7. Stankovic I, Belmans A, Prinz C, Ciarka A, Maria Daraban A, Kotrc M, et al. The association of volumetric response and longterm survival after cardiac resynchronization therapy. Eur Heart J Cardiovasc Imaging 2017;18:1109-17.

8. Haugaa KH, Edvardsen T, Smiseth OA. Mechanical dyssynchrony-resurrected as a flashing and rocking parameter to predict prognosis after cardiac resynchronization therapy. Eur Heart J Cardiovasc Imaging 2017;18:1118-9.

9. Zhou W, Garcia EV. Nuclear image-guided approaches for cardiac resynchronization therapy (CRT). Curr Cardiol Rep 2016;18:7.

10. Lancellotti P, Moonen M. Left ventricular dyssynchrony: A dynamic condition. Heart Fail Rev 2012;17:747-53.

11. Lafitte S, Bordachar P, Lafitte M, Garrigue S, Reuter S, Reant P, et al. Dynamic ventricular dyssynchrony: An exercise-echocardiography study. J Am Coll Cardiol 2006;47:2253-9.

12. Parsai C, Baltabaeva A, Anderson L, Chaparro M, Bijnens B, Sutherland GR. Low-dose dobutamine stress echo to quantify the degree of remodelling after cardiac resynchronization therapy. Eur Heart J 2009;30:950-8.

13. Chattopadhyay S, Alamgir MF, Nikitin NP, Fraser AG, Clark AL, Cleland JG. The effect of pharmacological stress on intraventricular dyssynchrony in left ventricular systolic dysfunction. Eur J Heart Fail 2008;10:412-20.

14. Stankovic I, Aarones M, Smith HJ, Voros G, Kongsgaard E, Neskovic AN, et al. Dynamic relationship of left-ventricular dyssynchrony and contractile reserve in patients undergoing cardiac resynchronization therapy. Eur Heart J 2014;35:48-55.

15. D'Andrea A, Mele D, Nistri S, Riegler L, Galderisi M, Agricola E, et al. The prognostic impact of dynamic ventricular dyssynchrony in patients with idiopathic dilated cardiomyopathy and narrow QRS. Eur Heart J Cardiovasc Imaging 2013;14:183-9.

16. Rocchi G, Bertini M, Biffi M, Ziacchi M, Biagini E, Gallelli I, et al. Exercise stress echocardiography is superior to rest echocardiography in predicting left ventricular reverse remodelling and functional improvement after cardiac resynchronization therapy. Eur Heart J 2009;30:89-97.

17. Ruschitzka F, Abraham WT, Singh JP, Bax JJ, Borer JS, Brugada $\mathrm{J}$, et al. Cardiac-resynchronization therapy in heart failure with a narrow QRS complex. N Engl J Med 2013;369:1395-405.

18. Legallois D, Marie PY, Franken PR, Djaballah W, Agostini D, Manrique A. Comparison of the dyssynchrony parameters recorded with gated SPECT in ischemic cardiomyopathy according to their repeatability at rest and to their ability to detect a synchrony reserve under dobutamine infusion. J Nucl Cardiol 2018.

19. Salimian S, Thibault B, Finnerty V, Gregoire J, Harel F. The effects of dobutamine stress on cardiac mechanical synchrony determined by phase analysis of gated SPECT myocardial perfusion imaging in a canine model. J Nucl Cardiol 2014;21:375-83.

20. Salimian S, Thibault B, Finnerty V, Gregoire J, Harel F. Phase analysis of gated blood pool SPECT for multiple stress testing assessments of ventricular mechanical dyssynchrony in a tachycardia-induced dilated cardiomyopathy canine model. J Nucl Cardiol 2017;24:145-57.

Publisher's Note Springer Nature remains neutral with regard to jurisdictional claims in published maps and institutional affiliations. 